\title{
Nose-cone calorimeter: PHENIX forward upgrade
}

\author{
Ondrej Chvala ${ }^{\mathrm{a}}$ \\ for the PHENIX Collaboration \\ University of California, Riverside, CA 92521, USA
}

Received: 30 September 2008 / Revised: 20 January 2009 / Published online: 28 February 2009

(C) Springer-Verlag / Società Italiana di Fisica 2009

\begin{abstract}
PHENIX is a high rate experiment efficient at measuring rare processes, but has limited acceptance in azimuth and pseudorapidity $(\eta)$. The Nose Cone Calorimeter (NCC), a W-Si sampling calorimeter in the region of $0.9<\eta<3$, is one of the upgrades which will significantly increase coverage in both azimuth and pseudorapidity. The NCC will expand PHENIX's precision measurements of electromagnetic probes in $\eta$, reconstruct jets, perform a wide scope of correlation measurements, and enhance triggering capabilities. The detector will significantly contribute to measurements of $\gamma$-jet correlations, quarkonia production, and low- $x$ nuclear structure functions. This report discusses details of the detector design and its performance concerning a sample of the physics topics which will benefit from the NCC. In view of recent funding difficulties, outlook of the activities is discussed.
\end{abstract}

PACS 29.40.Vj $\cdot 29.40 . \mathrm{Gx} \cdot 24.10 . \mathrm{Lx}$

\section{Introduction}

The PHENIX electromagnetic calorimeter (EMC) [1] covering the central rapidity region $0.35<|\eta|$, has been particularly important for various discoveries at RHIC, including suppression of light [2] and heavy [3] hadrons at high $p_{T}$, and direct $\gamma$ measurements [4].

The NCC [5-8] will expand the pseudorapidity coverage to $0.9<|\eta|<3$ in each arm, providing about ten times the rapidity coverage as the EMC, with enhanced $p_{T}$ range for neutral pions, and jet reconstruction. The NCC will lead to a better understanding of both hot and cold nuclear matter, and of the spin structure of the proton through measurements involving single hadrons, dihadrons, dileptons, direct photons, jets, and $\gamma$-jet correlations. Together with the muon arms, the NCC can reconstruct the $\chi_{C}$, allowing to study

a e-mail: chvala@bnl.gov temperature of the medium $[16,17]$. The rapidity coverage is suitable for low- $x$ and high $Q$ studies of both hot and cold nuclear matter, where saturation effects in gluon density are predicted by the Color Glass Condensate (CGC) [9-14] hypothesis. The NCC will also provide data for local level one (LL1) triggering, necessary at the RHIC-II luminosities.

This report discusses simulation efforts discussed in [15] for a single NCC arm, although a second arm would double the sensitivity and significantly aid correlation and jet studies. The failure of the review process presents a challenge for the collaboration, as the need for a calorimetry in the forward region is needed to take full advantage of other upgrades and RHIC-II luminosities.

\section{Design of the nose cone calorimeter}

The proposed NCC is a $\mathrm{Si}-\mathrm{W}$ calorimeter with tracking capabilities enabled by longitudinal and transversal segmentation. The NCC is $19 \mathrm{~cm}$ thick with a radius of $50 \mathrm{~cm}$, to be mounted on the vertex magnet coils, replacing the copper nosecone, as shown in Fig. 2.1.

The NCC composes of two finely segmented $(15 \times$ $15 \mathrm{~mm}^{2}$ ) sampling electromagnetic segments (EM1, EM2), and one coarse hadronic (HAD) segment. Tungsten was chosen for its high density $\left(19.3 \mathrm{~g} / \mathrm{cm}^{3}\right)$ and a small Molière radius. The depth of each EM segment is $8 X_{0}$ and $19 X_{0}$ for the HAD segment, totaling $35 X_{0}$ or 1.3 nuclear interaction lengths. Therefore EM showers are mostly extinct before they hit the HAD segment, allowing the measurement of EM probes in even the most central $\mathrm{Au}+\mathrm{Au}$ collisions. The design enables the distinction of electromagnetic from hadronic signals as shown in Fig. 2.2.

\subsection{High momentum $\pi^{0}$ identification}

The two EM showers from a high energy $\pi^{0}$ overlap on the tower level above energies of $\approx 5-7 \mathrm{GeV}$. These "single 
track" $\pi^{0}$ s are identified by a dedicated pre-shower module. The EM1 segment contains two layers of two perpendicular high resolution $\left(0.5 \times 60 \mathrm{~mm}^{2}\right)$ position sensitive Si strip layers (photon identifiers PI1 and PI2), placed at depths of 2 and $3 X_{0}$, as indicated by schematics in Fig. 2.2. The PI layers allow identification of $\pi^{0} \rightarrow \gamma \gamma$ reconstructing invariant mass using the spatial separation (opening angle) and energy distribution between the two hits in the PI layers. This enables $\pi^{0}$ identification up to $30 \mathrm{GeV}$ of momentum.

\subsection{Resolution of angle and energy measurement}

The tracking capability of the NCC is indicated in Fig. 2.3. In each segment, clusters are formed separately and their centers of gravity are calculated. These space points together with the interaction vertex point are fitted by a straight line to establish the original particle vector. Additional position information comes from PI sensors. The angular resolution

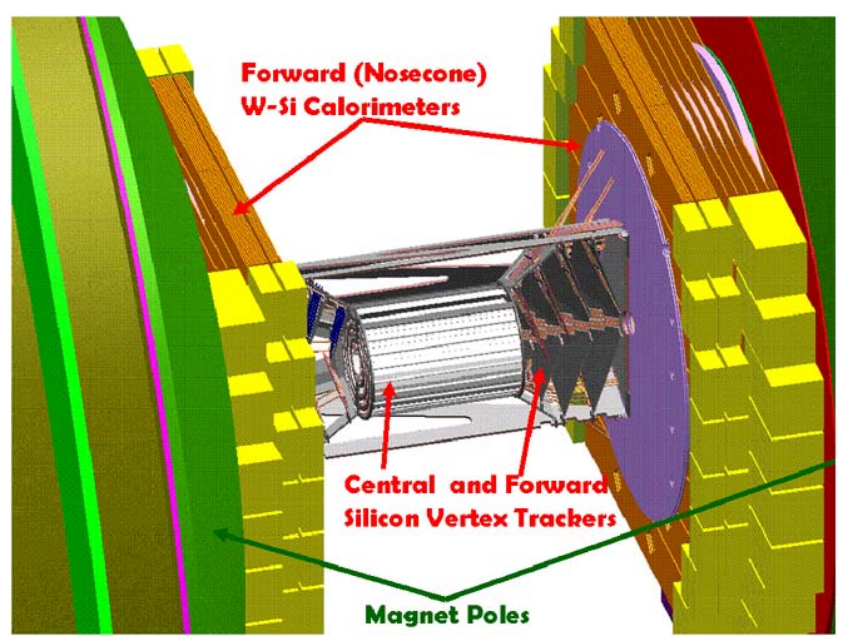

Fig. 2.1 Schematic diagram showing space negotiation of NCC and Silicon Vertex Trackers between the magnet poles of the NCC is $\approx 6 \mathrm{mrad}$ with the vertex position, and 10 to $18 \mathrm{mrad}$ depending on the impact angle without the vertex point.

Energy of a shower is calculated by applying the detector response function on the energies collected in all the segments separately, and summing the energies. The actual reconstruction algorithm shares energy deposits in towers and clusters among the reconstructed tracks using cluster shape parametrization and detector response function iteratively. The NCC energy resolution $\sigma_{E} / E$ is estimated as $18 / \sqrt{E[\mathrm{GeV}]}+4[\%]$, see Fig. 2.4 .

Total depth of EM1 and EM2 segments is $16 X_{0}$, therefore EM showers are mostly extinct before the HAD segment. This allows to measure EM probes even in the most central $\mathrm{Au}+\mathrm{Au}$ interactions with large hadronic background
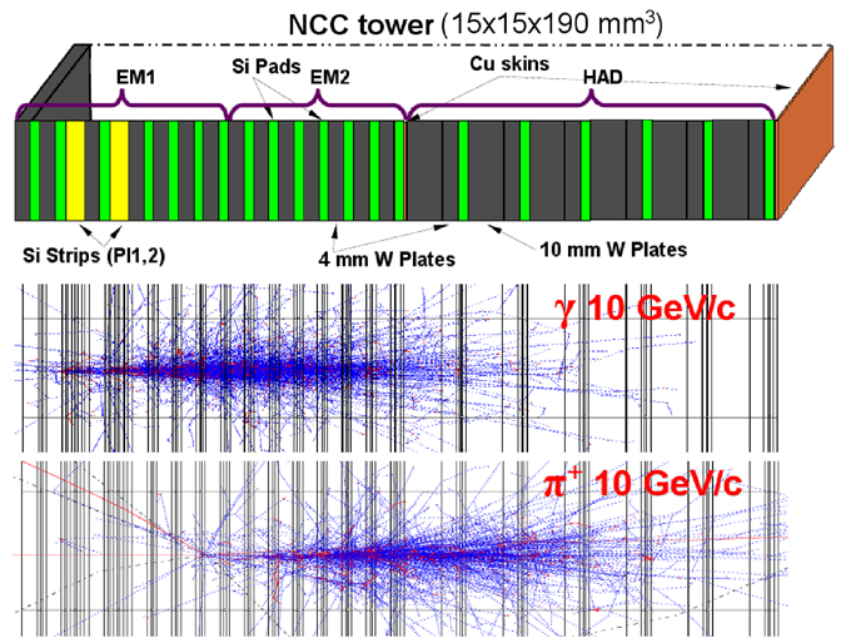

Fig. 2.2 Longitudinal structure of a single calorimeter tower showing the locations of the three calorimetric segments, EM1, EM2, and HAD, and the high-resolution position sensitive layers. Shown in the bottom panel are electromagnetic and hadronic showers due to a $10 \mathrm{GeV}$ photon and a charged pion of the same energy
Fig. 2.3 Principles of tracking measurements in the NCC. Linking the centers of gravity of the clusters found in each section provides tracking information without projective geometry, as illustrated

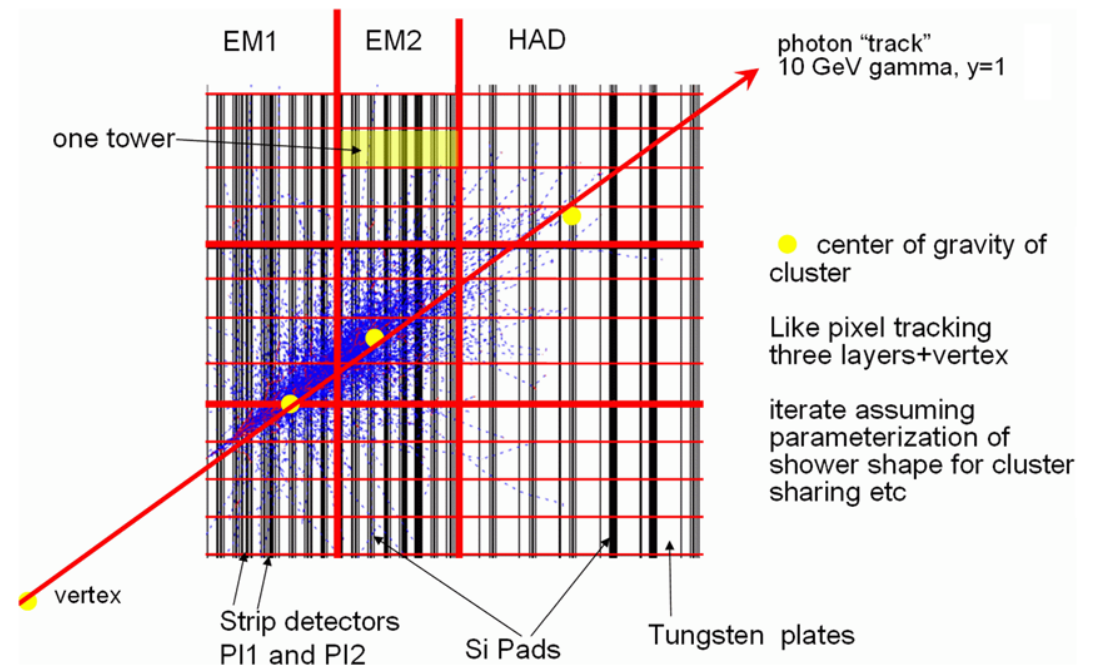




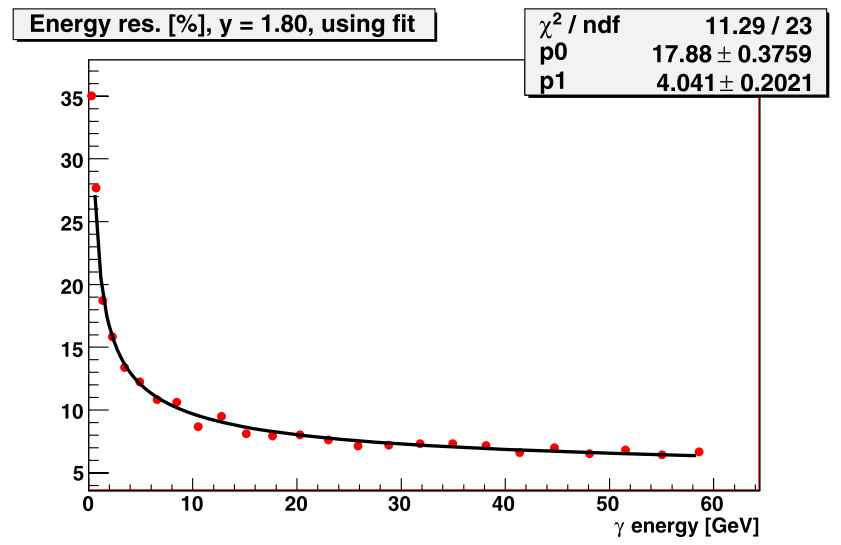

Fig. 2.4 Resolution of photon energy measurement [\%] at $\eta=1.8$, using EM1+EM2+HAD segments

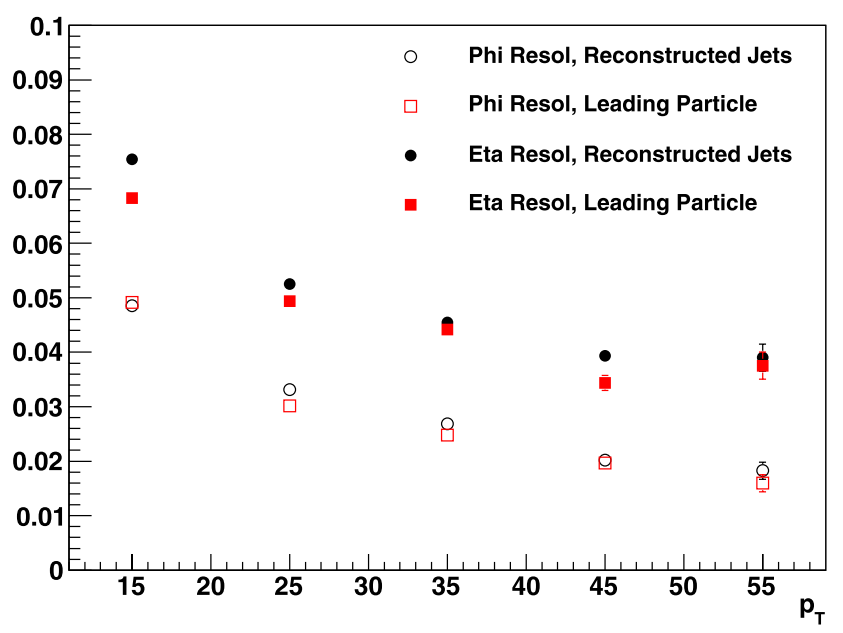

Fig. 2.5 Pointing resolution [rad] in $\varphi$ (open symbols) and $\theta$ (closed symbols) of NCC using leading particles (squares), and jets reconstructed using a cone algorithm (squares), PYTHIA
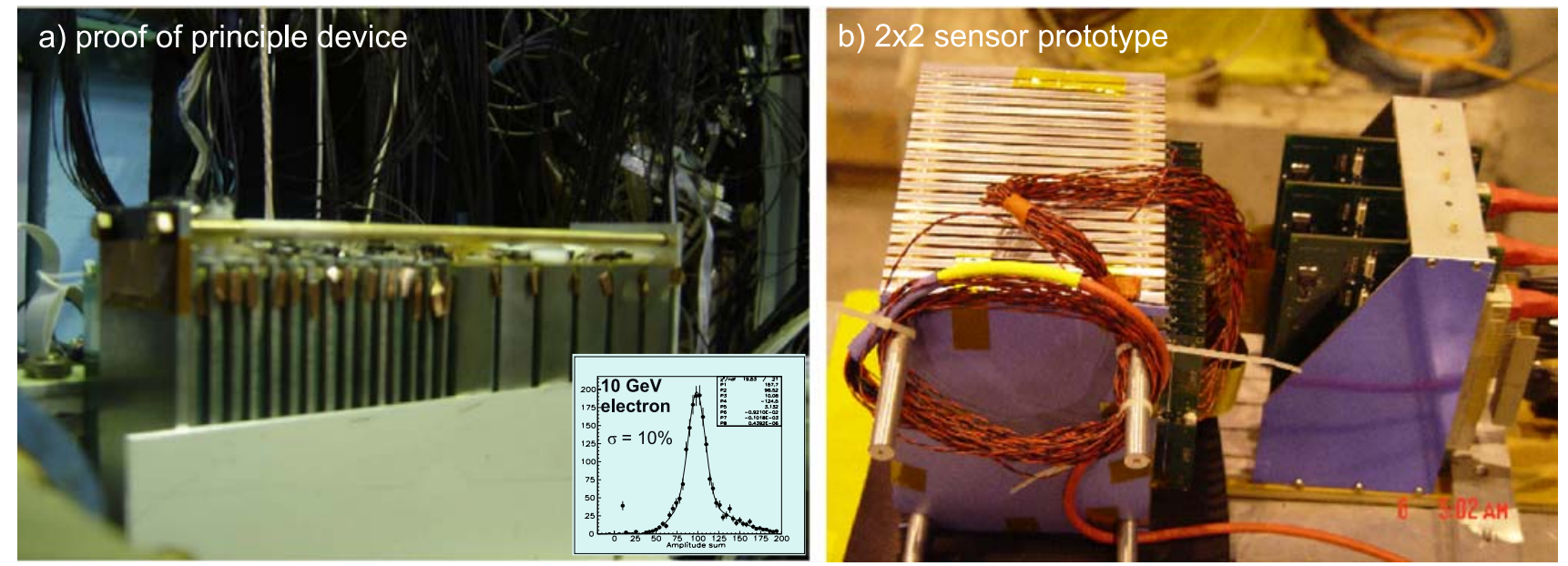

by using EM1 and EM2 deposits only, with only a small $\approx 0.5 \%$ loss in energy resolution stochastic term.

\subsection{Jets}

The HAD segment not only provides rejection of hadronic tracks for measurement of EM probes, it also improves hadronic energy resolution, aiding jet reconstruction. Energy resolution for jet reconstruction was estimated to be $\sigma_{E}^{\text {jet }} / E \approx 45 / \sqrt{E[\mathrm{GeV}]}[\%]$ via PYTHIA jets using a cone algorithm with $\mathrm{dR}<0.5 \mathrm{rad}$. The angular resolution determined from simulation is $\lesssim 0.1 \mathrm{rad}$, see Fig. 2.5. It contributes less than $10 \%$ to the $x$ resolution, below a value expected from NLO radiative effects.

\section{Prototypes}

Two NCC prototypes shown in Fig. 3.1 were constructed and tested. The first was used as a proof of principle and was used to test the design scheme and the robustness of the technology. It was exposed to $70 \mathrm{GeV} / c$ protons and $10 \mathrm{GeV} / c$ positrons at the IHEP U70 proton synchrotron in Protvino in Russia in November 2005, confirming that the design was sound and simulations describe the detector performance well; the insert in Fig. 3.1 shows $10 \%$ resolution $\sigma_{E}^{\text {jet }} / E$ for the $10 \mathrm{GeV}$ electrons.

The second prototype was a $2 \times 2$ sensor module using the final stackable design, tower geometry, materials, and read-out electronics. It was used to test the design for the PI layers and the performance of the electronics. Tests were performed in September 2007 at CERN using electron beams at a range of energies up to $100 \mathrm{GeV}$.

Third prototype is currently under construction. It will correspond to the smallest NCC brick, $16 \times 8$ towers large.

Fig. 3.1 NCC prototypes: (a) 2005 proof of principle, (b) 2007 final geometry 
After a scheduled test beam at Fermilab in early 2009, the prototype is envisaged to be integrated into the PHENIX experiment for Run 9 or 10 in a temporary location.

\section{Simulations of detector performance}

The NCC was modeled in GEANT-3, both as a stand-alone detector and integrated into the PHENIX Integrated Simulation Application (PISA) framework. The stand-alone simulation was used to establish the detector sampling fractions, a parameterization of EM shower shape, and energy calibration.

The PISA simulation includes realistic descriptions of all components of the PHENIX detector and was used to estimate the sensitivity of the NCC to $\pi^{0} \mathrm{~s}$, single photons, and $\chi_{C} \mathrm{~s}$, expressed as a $\mathrm{R}_{A A}$ ratios. ${ }^{1}$

The simulations were performed in a standard fashion by merging single particle per event signal files with HIJING v1.37 generated background events. The backgrounds were $\mathrm{p}+\mathrm{p}$ minbias, $\mathrm{d}+\mathrm{Au}$ minbias, $\mathrm{d}+\mathrm{Au}$ central $10 \%$, and 10 centrality bins in $\mathrm{Au}+\mathrm{Au}$ interactions, each corresponding to an established centrality class used in PHENIX data analyses. All the collisions were simulated at a center of mass energy of $200 \mathrm{GeV}$.

The sensitivity studies aimed at one ten weeks long run at RHIC-II luminosities. Since the computing time and space requirement to simulate the full RHIC-II luminosity is prohibitive, smaller event samples of about $50 \mathrm{k}$ events in each centrality class were processed, then scaled up to a full run, using the signal to background and efficiency factors found by the simulation, reconstruction and analysis chain.

\section{$4.1 \pi^{0}$ analysis}

Two techniques are used to reconstruct $\pi^{0} \mathrm{~s}$. For energies below about $5 \mathrm{GeV}$, the standard method of forming the invariant mass of all pairs of photons with energy greater than $0.5 \mathrm{GeV}$ is used. We call these "two-track" $\pi^{0} \mathrm{~s}$. Cuts on the longitudinal and lateral $\chi^{2}$ were imposed on each photon. The opening angle between the two photons was required to be greater than $50 \mathrm{mrad}$ and the energy asymmetry was required to be less than 0.8 . Background is subtracted by event mixing. High detector occupancy in the most central $\mathrm{Au}+\mathrm{Au}$ collisions limits the rapidity acceptance below 1.5 in this analysis.

For higher energy $\pi^{0} \mathrm{~s}$ the two photons overlap. In order to identify such showers as $\pi^{0}$ candidates, the strip detectors PI1 and PI2 were used to obtain an opening angle and energy asymmetry. This information together with the total energy

${ }^{1}$ For brevity of the report the result of spin studies at $\sqrt{s}$ of $500 \mathrm{GeV}$ included in the DoE review report [15] are omitted.

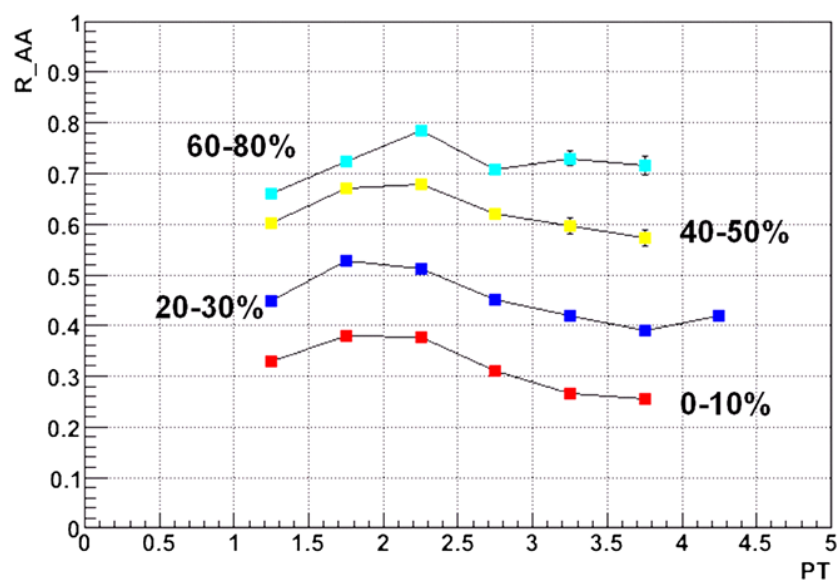

Fig. 4.1 $\mathrm{R}_{A A}$ for $\pi^{0} \rightarrow \gamma+\gamma$ for various values of centrality. The value of $\mathrm{R}_{A A}$ is assumed to be the same as that seen in the central arms, the error bars reflect the precision possible from one run at RHIC II luminosities. The $p_{T}$ reached is about $4 \mathrm{GeV}$. Above this $p_{T}$, the single track $\pi^{0}$ takes over

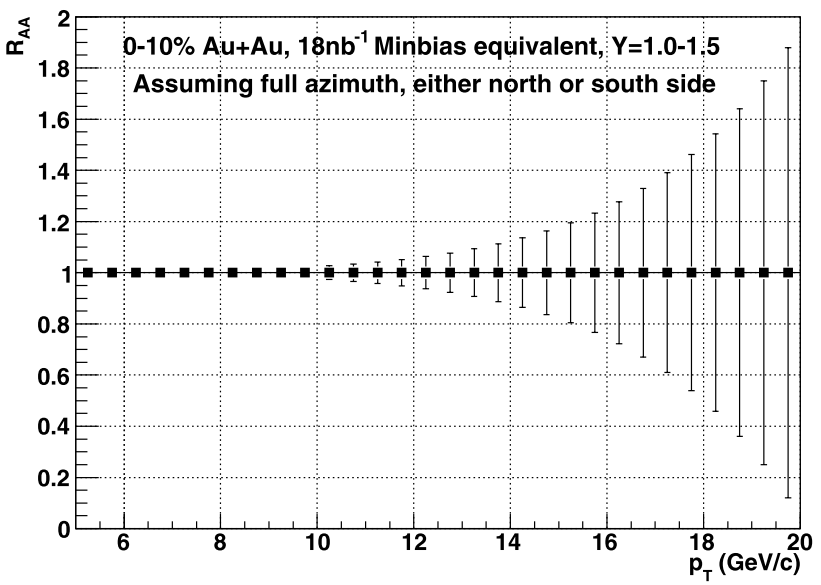

Fig. 4.2 $\mathrm{R}_{A A}$ for "single track" $\pi^{0}$ 's. The error bars reflect the precision possible in one run at RHIC II luminosities. We assume a suppression pattern similar to that seen in the central arms, hence statistics runs out at $\sim 15 \mathrm{GeV}$

in the shower was used to form an invariant mass. A cut of $135 \pm 50 \mathrm{MeV}$ is applied to the resulting mass to select $\pi^{0}$ candidates; these are referred to as "single track" $\pi^{0}$ s. Each candidate is assigned a weight giving the probability that it is a $\pi^{0}$.

Estimates for the uncertainties on the determination of $\mathrm{R}_{A A}$ using a two-track reconstruction of the $\pi^{0}$ at relatively low $p_{T}$ are shown in Fig. 4.1; and at higher $p_{T}$ in Fig. 4.2 using the method of single-track $\pi^{0}$ reconstruction.

\subsection{Direct photon analysis}

Direct photons are defined as those photons that do not come from final state hadron decays. In high multiplicity heavy ion collisions, direct photons can only be extracted 

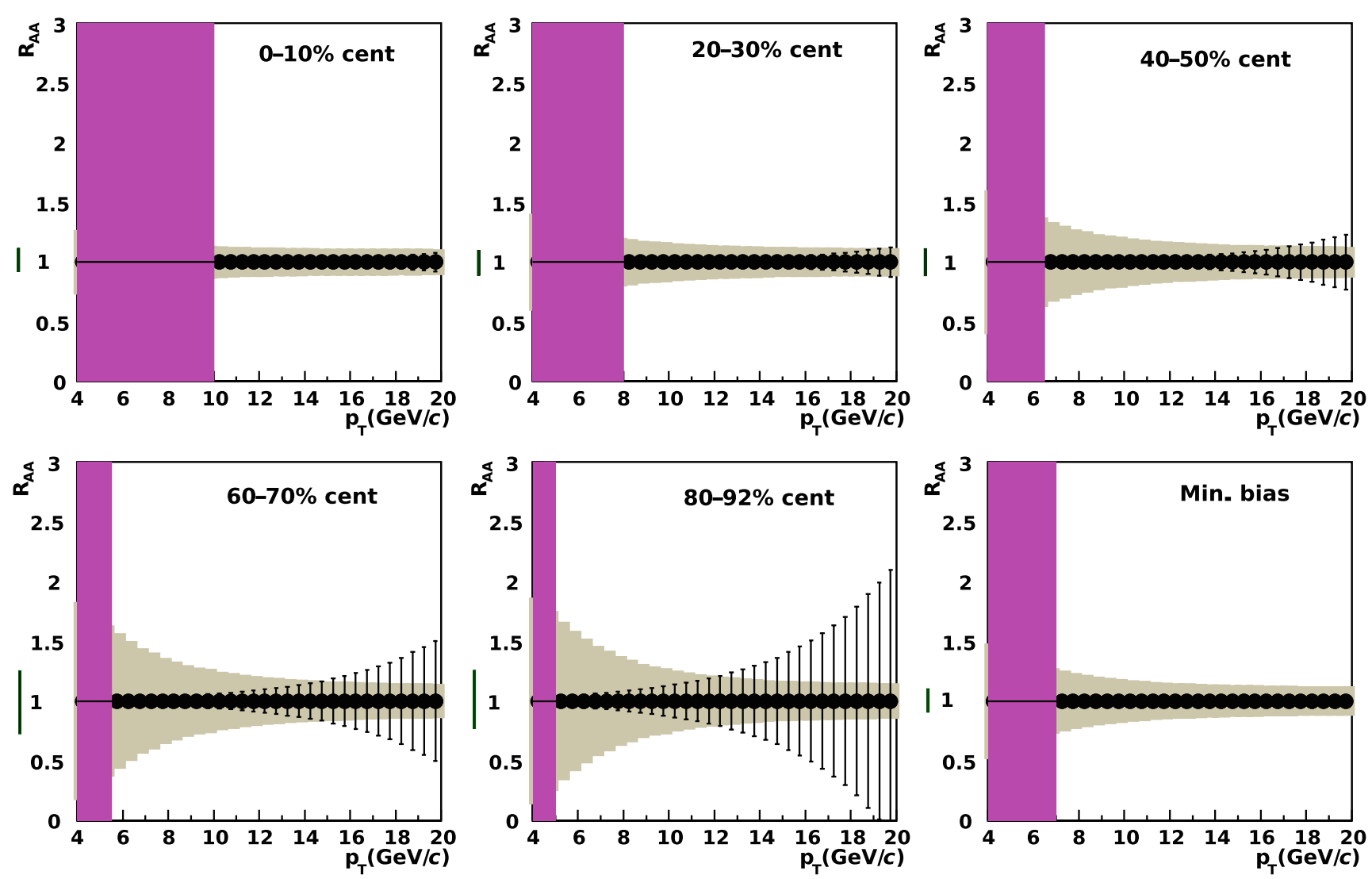

Fig. 4.3 Anticipated sensitivity for direct photon $\mathrm{R}_{A A}$ in several centralities $\mathrm{Au}+\mathrm{Au}$ after one RHIC-II run

by first measuring the spectra of inclusive photons, then subtracting contributions from hadron decays. Measurements of hadrons which decay into photons are used to simulate the contribution from decays.

Below $5 \mathrm{GeV}$ the direct photon signal is a few percent of the total photon spectrum in $\mathrm{p}+\mathrm{p}$, and less than $10-20 \%$ in central $\mathrm{Au}+\mathrm{Au}$. However above $5 \mathrm{GeV}$ the signal to background (S/B) rises quickly, reaching unity in central $\mathrm{Au}+\mathrm{Au}$ collisions already around $10 \mathrm{GeV}$, largely due to the factor of 5 suppression of $\pi^{0}$ at mid-rapidity [2]. The increased S/B is concurrent with increased occupancy and overlap probability in the NCC. The expected sensitivity of the direct photon measurement is shown in Fig. 4.3. Solid and shaded error bars indicate statistical and systematical uncertainties; shaded areas at low $p_{T}$ indicate regions where the current method gives ambiguous results. [15] discusses and enumerates the various sources of the errors in detail.

\section{$4.3 \chi_{C}$ analysis}

The NCC will allow PHENIX to measure the $\chi_{C}$ through its decay $\mathrm{J} / \psi+\gamma$, where the $\mathrm{J} / \psi \rightarrow \mu \mu$ is detected in the muon arms. When both muons are detected in the muon arms, about $60 \%$ of the photons are within the NCC acceptance. Dimuons are identified using the standard proce-

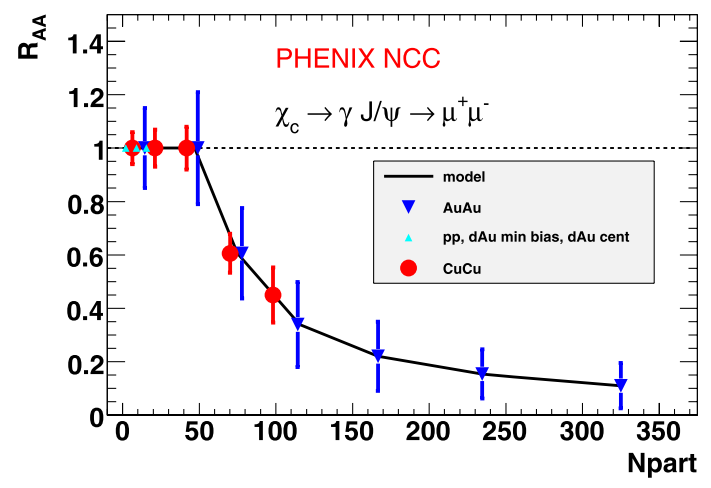

Fig. 4.4 Anticipated sensitivity for $\chi_{C} \mathrm{R}_{A A}$ in $\mathrm{Au}+\mathrm{Au}$ after one RHIC-II run

dure [18]. Photons are identified using a longitudinal $\chi^{2}$ cut, and requiring a shower energy above $300 \mathrm{MeV}$. Figure 4.4 shows the significance expected for the $\mathrm{R}_{A A}$ of the $\chi_{C}$ within $1<\eta<1.5$. The $\mathrm{S} / \mathrm{B}$ for the most central $\mathrm{Au}+\mathrm{Au}$ events used in this simulation is $1 / 400$. Note that in another analysis, PHENIX has successfully identified signals using a mixed background subtraction with a S/B of less than 1/1000. Therefore, using the NCC, PHENIX will be able to make an excellent measurement of the suppression factor for the $\chi_{C}$. 


\section{Future of the NCC project}

The collaboration was charged with extraction of several physics observables from a full detector simulation with realistic backgrounds and one RHIC-II run luminosity, by a DoE review committee. Part of the results of this effort [15] are summarized in the presented article. Unfortunately the review process was not a success, limiting funding of the full scope of the project in the time scale compatible with other RHIC-II detector and machine upgrades.

Extension of PHENIX calorimetry phase space coverage is however necessary for the RHIC-II physics program, and the collaboration is determined to solve the issue. Also, other groups expressed interest in $\mathrm{Si}-\mathrm{W}$ calorimetry for experiments such as ILC.

Several proposals are currently studied and investigated intensively, including other funding resources from interested parties and cutting the NCC cost. Options include building only about $1 / 4$ of the NCC azimuthal coverage and eliminate HAD segment, which would still allow for measurements in $\mathrm{p}+\mathrm{p}$ and $\mathrm{d}+\mathrm{A}$ collisions; as the largest contribution to the total detector cost comprise the Si pads, a hybrid option combining a $2 X_{0}$ Si-based module with 4 pads and PI layers, with a scintillator based readout.

\section{Conclusions}

The Nose-Cone Calorimeter (NCC) is a next generation $\mathrm{Si}-\mathrm{W}$ sampling calorimeter with tracking capabilities, providing rapidity coverage about 10 times that of the central arms. The detector material is the densest with the smallest Molière radius affordable on a reasonable budget.

The NCC will provide precision measurement of electromagnetic probes in forward rapidity region between 0.9 and 3 including $\pi^{0} \mathrm{~s}$ up to $30 \mathrm{GeV}, \gamma /$ hadron discrimination via longitudinal shower shape measurement, jet reconstruction, and the data for fast local level one triggering.

This upgrade will significantly enhance PHENIX detector system ability to measure properties of sQGP medium, namely suppression of hadrons in forward direction, medium temperature using charmonium suppression, and opacity of the medium by $\gamma$-jet correlations. In $\mathrm{d}+\mathrm{Au}$ collisions the $\mathrm{NCC}$ will uncover the structure of very high density nuclear matter, in particular the saturation effect down to low- $x$ as a precursion to sQGP. The gluon and the sea polarization in a proton at low- $x$ is the main aim of NCC measurement with respect to the RHIC spin program.

Despite the recent setback, several options of how to arrive to PHENIX forward calorimetry coverage in RHIC-II era are being investigated.

\section{References}

1. K. Adcox et al., Nucl. Instrum. Methods A 499, 469 (2003)

2. A. Adare et al. (PHENIX Collaboration), Phys. Rev. Lett. 101, 232301 (2008). arXiv:0801.4020

3. A. Adare et al. (PHENIX Collaboration), Phys. Rev. Lett. 98, 172301 (2007). arXiv:nucl-ex/0611018

4. A. Adare et al. (PHENIX Collaboration), Phys. Rev. Lett. (submitted). arXiv:0804.4168

5. PHENIX Collaboration, PROPOSAL for a Nosecone Calorimeter (NCC) for the PHENIX Experiment www.phenix.bnl.gov/ phenix/WWW/docs/upgrades/ncc/PHENIX_NCC_march2006. pdf

6. E. Kistenev et al., IEEE Nucl. Sci. Symp. Conf. Rec. 1, 591 (2004)

7. V. Boncvicini et al., IEEE Trans. Nucl. Sci. 52(4), 874 (2005)

8. E. Kistenev, Czechoslov. J. Phys. 55, 1659 (2005)

9. L. McLerran, R. Venugopalan, Phys. Rev. D 49, 2233 (1994)

10. L. McLerran, R. Venugopalan, Phys. Rev. D 49, 3352 (1994)

11. L. McLerran, R. Venugopalan, Phys. Rev. D 50, 2225 (1994)

12. E. Iancu, A. Leonidov, L. McLerran, arXiv:hep-ph/0202270

13. E. Iancu, A. Leonidov, L. McLerran, arXiv:hep-ph/0202025

14. R. Seto, Proc. of Workshop on Nuclear Dynamics, Bahamas, January 2002, World Scientific (2002). arXiv:nucl-ex/0204003

15. PHENIX Collaboration, Physics and Simulations; in response to the DOE review; for a Nosecone Calorimeter (NCC); for the PHENIX Experiment http://www.phenix.bnl.gov/phenix/WWW/ publish/seto/seto/ncc/TaskForceDocs/NCCresponse.pdf

16. A. Mocsy, P. Petreczky, Phys. Rev. Lett. 99, 211602 (2007). arXiv:0706.2183

17. A. Mocsy, P. Petreczky, Phys. Rev. D 77, 014501 (2008). arXiv:0705.2559

18. A. Adare et al. (PHENIX Collaboration), Phys. Rev. Lett. 98, 232301 (2007). arXiv:nucl-ex/0611020 